\title{
CARCINOME SPINOCELLULAIRE DE LA LEVRE INFERIEURE SUR CICATRICE DE LUPUS ERYTHEMATEUX CHRONIQUE
}

\author{
A. LACHKHEM , W. ABID , A. CHORFA ,R. BACHRAOUI ,K. KHAMMASSI , S. TOUATI , S. GRITLI \\ SERVICE DE CHIRURGIE CARCINOLOGIQUE, ORL ET CERVICO-FACIALE \\ INSTITUT SALAH AZAÏEZ. TUNIS.
}

\begin{abstract}
RESUME
Le carcinome spinocellulaire est une tumeur invasive développée aux dépens des kératinocytes épidermiques. II survient plutôt sur des lésions de dysplasies.

Nous rapportons le cas d'un homme de 35 ans, connu porteur depuis l'âge de 15 ans de lupus discoïde chronique, non traité.

Ce patient nous a consulté pour une lésion bourgeonnante de l'hémi lèvre inférieure gauche évoluant depuis une année, apparue sur une lésion lupique.

La biopsie a conclu à un carcinome spinocellulaire.

Le patient a eu une exérèse tumorale avec un curage triangulaire homolatéral et une reconstruction en un seul temps. Mots-clé : Carcinome spinocellulaire, carcinome épidermoïde, lupus discoïde chronique, dermatose pré-cancéreuse.
\end{abstract}

Epidermoïd carcinoma is the most common malignant tumor of the lips.

They often develop on precancerous lesions. Leukoplasia is the most frequent precancerous lesion.

We report a case of a 35 years old man, who suffered from discoid lupus erythematous since 20 years; and wasn't treated from.

He consulted for a squamous ulceration of the under lip, developed from one year.

The biopsy concluded to an epidermoïd carcinoma.

He was treated with surgical excision with reconstruction and homolateral triangular recovery.

Keywords: Squamous cell carcinoma, epidermoïd carcinoma, discoid lupus erythematosus, precancerous lesion.

\section{INTRODUCTION}

Les carcinomes spinocellulaires ou carcinomes épidermoïdes sont des tumeurs fréquentes, invasives, développées aux dépens des kératinocytes épidermiques.

On les trouve surtout sur des zones photo-exposées, ils surviennent plutôt sur des lésions de dysplasies.

Ils sont en général de bon pronostic quand l'exérèse chirurgicale est effectuée rapidement avec une marge de tissu sain suffisante.

Leur prévention repose sur la photoprotection et la surveillance dermatologique des sujets à risque.

\section{OBSERVATION}

Nous rapportons le cas d'un homme de phénotype clair, âgé de 35 ans journalier, tabagique à 40 paquets-années, ayant une exposition aux UVA, aux antécédents de lupus érythémateux discoïde du visage et du décolleté avec photosensibilité négligé par le patient et non traité, évoluant depuis plus de 20 ans.

II a présenté une lésion de l'hémilèvre inférieure gauche, évoluant depuis une année, augmentant progressivement de volume.

L'examen clinique retrouve une lésion ulcéro-bourgeonnante de la lèvre rouge inférieure latéralisée à gauche de
$2 \mathrm{~cm}$ de grand axe restant à distance de la ligne médiane sur une plaque érythémato-squameuse (Fig.1) . Les gencives sont saines ainsi que le sillon gingivolabial.

Nous avons trouvé à l'examen cervical une adénopathie homolatérale de $1 \mathrm{~cm}$ du secteur $\mathrm{llb}$, ferme, ronde, mobile, indolore, peau en regard saine.

Par ailleurs, il présente des plaques érythématosquameuses avec atrophie centrale et une cicatrice atrophique dépigmentée au niveau de la lèvre supérieure et inférieure, du pavillon de l'oreille et du décolleté d'une façon symétrique (Fig.2).

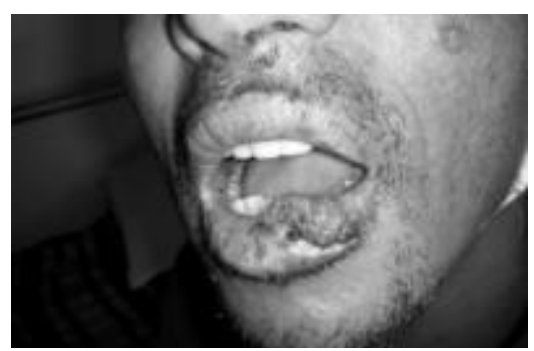

Fig. 1 : Lésion bourgeonnante de la lèvre rouge inférieure. 


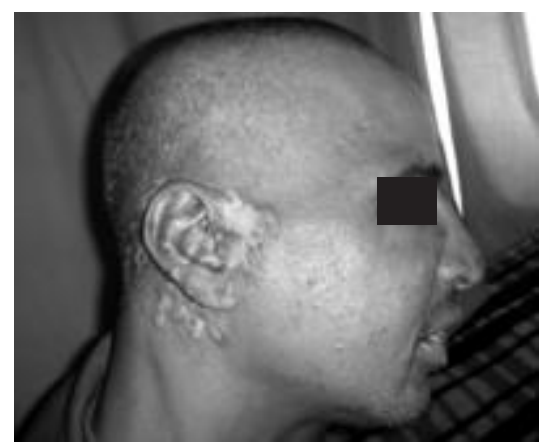

Fig.2 : Plaques érythémato-squameuses avec atrophie centrale et cicatrice atrophique dépigmentée

Après un bilan d'extension, la tumeur était classée : T1N1M0.

La décision thérapeutique a été prise au sein d'un comité médical multidisciplinaire. II a eu une exérèse tumorale et réparation en $\mathrm{W}$ avec un curage triangulaire homolatéral du secteur I, II et III (Fig. 3).

Les suites opératoires étaient simples.

Le recul est de 1 an sans récidive (Fig. 4).
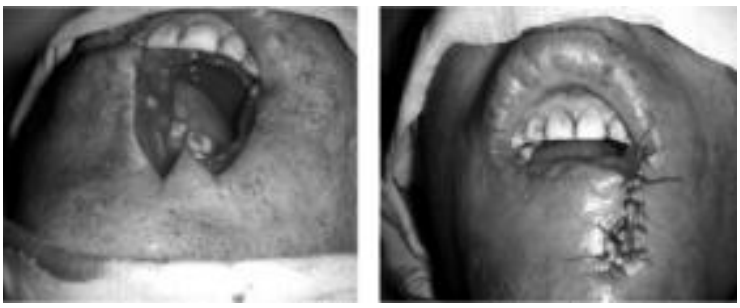

Fig.3 : Exérèse tumorale et réparation en W

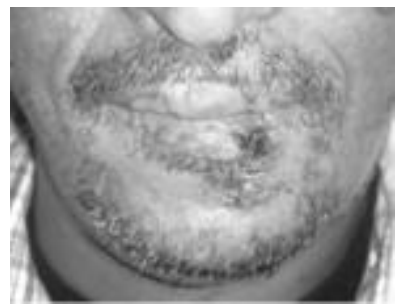

Fig.4 : Aspect post-opératoire

\section{DISCUSSION}

Le cancer de la lèvre paraît se rapprocher sur le plan étiologique du cancer de la peau (1). II représente $12 \%$ des cancres de la tête et du cou (2).

Le carcinome épidermoïde est le plus fréquent $(90 \%$ des cas) des cancers des lèvres (3), et représente $50 \%$ des carcinomes épidermoïde de la face (4).

II siège souvent sur le tiers moyen de la lèvre inférieure $(3,4)$

C'est l'homme de plus de 60 ans qui est le plus souvent touché, le sex-ratio étant de six à neuf.

Le carcinome épidermoïde survient souvent sur des lésions précancéreuses cutanées tel que le lupus érythémateux chronique (LEC), qui est une dermatose faite de plaques érythémato-squameuses avec atrophie centrale qui peut évoluer vers une cicatrice atrophique dépigmentée.

La survenue d'un carcinome spinocellulaire de la lèvre inférieure est possible sur des lésions de chéilite chronique dues au LEC ; qui sont des hyperkératoses réactionnelles apparaissant sur des lésions initialement érythémateuses et squameuses à surface irrégulière non indurée.

Les chéilites actiniques s'observent surtout chez les hommes de plus de 45 ans, exposés par leur profession au soleil et aux intempéries pendant plusieurs années (agriculteurs, montagnards, marins), d'autant plus que leur phototype est plus clair (blonds ou roux).

Les chéilites actiniques atteignent plus fréquemment la lèvre inférieure, plus exposée aux rayons ultraviolets. Ces derniers entraînent des altérations de I'ADN des kératinocytes basaux et une diminution de leur activité mitotique.

L'épithélium devient à terme atrophique et la capacité photoprotectrice est diminuée.

Les femmes seraient relativement protégées par l'utilisation de cosmétiques labiaux. Le traitement au laser $\mathrm{CO} 2$ vise à améliorer l'apparence de la lèvre et à éviter que les lésions ne deviennent cancéreuses.

Une mauvaise hygiène buccodentaire et surtout l'intoxication tabagique associée aggravent classiquement l'évolution des lésions.

A un stade tardif, des dysplasies épidermiques peuvent apparaître ou bien des carcinomes épidermoïde s; ce qui impose une surveillance régulière de ces lésions de chéilite actinique (au moins 1 fois par an).

L'étude de Bernastsky et al $(5,6)$ démontre un risque relatif de cancers de 1,15 chez les lupiques.

La fréquence des cancers cutanés sur lésion de L.E.C est estimée à $2 \%$, et dans $95 \%$ des cas il s'agit d'un carcinome spinocellulaire.

Les facteurs incriminés dans la genèse de cette association morbide sont nombreux et variés et on peut citer comme exemple (7):

- les irradiations, l'arsenic autrefois utilisé dans le traitement du LEC

- les perturbations immunitaires : la diminution des lymphocytes "T" suppresseurs et le rôle cytotoxique des anticorps anti-nucléaires,

- le caractère cicatriciel de la lésion (rôle essentiel)

- l'effet dépigmentant du L.E.C majore la photosensibilité. Notre patient présente comme facteur de risque une exposition chronique aux UVA, l'absence de protection solaire et l'aspect dépigmentant des lésions du lupus discoïde. 
La lèvre inférieure est à peu près le seul endroit où le lupus érythémateux photosensible peut donner lieu à une transformation carcinomateuse. Le risque augmente avec la durée de l'exposition aux UV (avec un effet seuil) et avec l'âge.

Le délai d'apparition de la tumeur est de 7 à 20 ans après l'apparition du L.E.C (7).

Le délai d'apparition de la tumeur chez notre patient était de 20 ans après l'apparition du lupus discoïde.

Le traitement de ces types de cancers consiste en une chirurgie d'exérèse large avec reconstruction. Dans notre cas le patient a eu une exérèse tumorale et réparation en W.

L'aspect fonctionnel est un élément primordial de toute reconstruction labiale avec respect de l'équilibre statique et dynamique (8).

D'après la série d'Ezzoubi et al (9), le résultat thérapeutique fonctionnel est jugé satisfaisant dans $92 \%$ des cas opérés. Le résultat esthétique est jugé excellent dans 60 $\%$ des cas et bon dans $30 \%$ des cas. Le recul moyen est de 2 ans, et la survie à 2 ans est de $90 \%$.

D' après la série de Poncet et al (1), la survie brute des patients traités d'un carcinome épidermoïde de la lèvre était de $93 \%$ à 1 an, de $81 \%$ à 3 ans et de $70 \%$ à 5 ans. La survie relative à 5 ans était de $96 \%$ pour les hommes et de $88 \%$ pour les femmes

\section{CONCLUSION}

Le carcinome épidermoïde est la tumeur maligne la plus fréquente des lèvres. Les causes essentielles sont l'exposition solaire, le tabagisme et les irritations chroniques.
Les lésions cutanées du L.E.C représentent une lésion précancéreuse des carcinomes épidermoïdes. Même si le risque de dégénérescence est minime, ces lésions doivent être surveillées, surtout dans notre pays où l'ensoleillement est particulièrement important. Le traitement est la chirurgie d'exérèse avec plastie de reconstruction, associée a une éviction des facteurs aggravants comme le tabac.

\section{REFERENCES}

1-Diombana M.L. Tumeurs de la lèvre et des joues au service de stomatologie de l'hôpital national de Kati (Mali), a propos de 44 cas. Médecine d'Afrique Noire. $1996 ; 43: 8-9$.

2- Perrinaud A. Carcinomes épidermoïdes (ou spinocellulaires). Presse Med. 2008; 37: 1485-1489.

3-Ben Slama L. Carcinome des lèvres. Presse Med. 2008 ; 37: 1490-1496.

4-MS Gautier. Tumeurs cutanées avec lésions précancéreuses. AKOS Encyclopédie Pratique de Médecine ; 2-0724.

5-Bernatsky S, Boivin JF, Joseph L, Rajan R, Zoma A, Manzi S. An international cohort study of cancer in systemic lupus erythematosus. Arthritis Rheumatism 2005;52:1481-90.
6-Broussolle C., Seve P. Cancers au cours des maladies auto-immunes. La revue de médecine interne. $2006 ; 27: 277-280$

7-B.Hassem, K.Senouci, B. Lazrak, F.Bennouna-Biaz. Carcinomes épidermoïdes de la lèvre inférieure sur cicatrice de lupus erythémateux chronique. Médecine du Maghreb. $1991 ; 28$

8-Brix M. Principes généraux de la chirurgie des lèvres. Annales de Chirurgie Plastique Esthétique. $2002 ; 47: 413-422$

9 -Ezzoubi M. La reconstruction après exérèse carcinologique des cancers des lèvres. A propos de 100 cas. Revue de laryngologie d'otologie et de rhinologie. 2005; 126(3):141-6. 